\title{
Novel polymorphisms within the DIk1-Dio3 imprinted locus in rat: a putative genetic basis for strain-specific allelic gene expression
}

\section{Laura J. Sittig ${ }^{\dagger}$ and Eva E. Redei*}

Department of Psychiatry and Behavioral Sciences, The Asher Center, Feinberg School of Medicine, Northwestern University, Chicago, IL, USA

\section{Edited by:}

Bob Kesterson, University of

Alabama at Birmingham, USA

Reviewed by:

Margit Burmeister, University of

Michigan, USA

Michael P. McDonald, University of

Tennessee Health Science Center,

USA

\section{*Correspondence:}

Eva E. Redei, David Lawrence Stein Professor of Psychiatry, Department of Psychiatry and Behavioral

Sciences, Feinberg School of Medicine, Northwestern University, 303 E. Chicago Ave 9-223, Chicago, IL 60611, USA.

e-mail: e-redei@northwestern.edu

${ }^{\dagger}$ Present address::

Laura J. Sittig, Department of Human Genetics, University of Chicago, 920 E. 58th Street CLSC 501, Chicago, IL 60637, USA.
The imprinted iodothyronine deiodinase-III (Dio3) thyroid hormone metabolizing gene exhibits paternal expression in most fetal tissues, yet exhibits aberrant, maternal expression in the hippocampus in F1 offspring of Sprague Dawley (SD) $\times$ Brown Norway (BN) rats. The maternal hippocampal expression is associated with lower Dio3 mRNA levels specifically in the hippocampus. Here, we tested the hypothesis that genetic polymorphisms between the SD and BN parent strains cause this aberrant allelic Dio3 expression and contribute to behavioral sequelae of higher thyroid hormone levels locally in the hippocampus, including anxiety-related behavior. We mapped and sequenced the Dio3 gene and several previously unmapped regions in the DIk1-Dio3 locus that could regulate imprinting of the Dio3 gene. In the Dio3 promoter we identified four novel polymorphisms between the BN and SD strains. Next we took advantage of the fact that the Long Evans (LE) strain exhibits identical polymorphisms as the SD strain in the region $5^{\prime}$ and including the Dio3 gene. By reciprocally crossing LE and BN strains we tested the relationship among Dio3 promoter region polymorphisms and Dio3 mRNA expression in the hippocampus. Aberrant strain-specific hippocampal Dio3 allelic expression replicated in the LE-BN reciprocal crosses, suggesting that hippocampal-specific imprinting of the Dio3 gene is not the result of a unique genetic or epigenetic characteristic of the SD rat strain, or a unique epistatic interaction between SD and BN. To our knowledge no other studies have reported a genetic $\times$ epigenetic interaction of genetic origin in the brain.

Keywords: hippocampus, iodothyronine deiodinase-III, allelic expression, imprinting, parent-of-origin, rat, polymorphism

\section{INTRODUCTION}

Parent-of-origin allele-specific gene expression, often called genetic imprinting in its complete form, is enriched in the brain. In fact, widespread brain region-specific allelic expression has been reported (Gregg et al., 2010) [but see Deveale et al. (2012)], and has been hypothesized to be related to the different functions served by different regions of the brain. Examples of neurodevelopmental disorders resulting from genetic mutations in imprinted genes or imprinting regulatory regions include Prader-Willi, Angelman, and Rett syndromes (Davies et al., 2005; Kernohan and Berube, 2010), yet it is not known how or if brain region-specific allelic expression plays into these disorders. Apart from overt syndromes, it is possible that genetic differences in imprinted genes or imprinting regulatory regions that do not necessarily cause disease could differently affect the function of specific brain regions and behavioral vulnerability. Defining and understanding such interactions will be increasingly important toward understanding the normal brain and neuropsychiatric disorders.

In recent studies, we identified one example of brain regionspecific, parent-of-origin specific allelic expression of the gene encoding the thyroid hormone metabolizing enzyme, Dio3. We crossed two rat strains [Sprague Dawley (SD), S and
Brown Norway (BN), B] to produce reciprocal crosses, abbreviated SB and BS with maternal strain indicated first. The Dio3 gene showed preferential paternal expression in the fetal brain and biallelic expression in adult brain of the BS offspring similarly to the expression profile reported previously (Edwards et al., 2008; Gregg et al., 2010). However, the SB offspring showed a preferential maternal Dio3 expression in hippocampus that was present in both fetal and adult brain, and corresponded to lower total expression of Dio3 mRNA. The resulting sequelae included a higher level of T3 in the hippocampus (as the Dio3 enzyme is responsible for inactivating thyroid hormones) and several anxiety-related behaviors (Sittig et al., 2011a). The relationship between hippocampal Dio3 expression, lower total Dio3, elevated T3 in hippocampus, and anxiety-related deficits was substantiated in a separate study using SB-BS reciprocal crosses, in which the SB difference in hippocampal Dio3 expression was exacerbated by exposure to prenatal alcohol, producing further elevation in hippocampal T3 levels and behavioral deficits (Sittig et al., 2011b). Here, we aim to confirm a genetic cause for aberrant hippocampal Dio3 allelic expression, show candidate causative polymorphisms, and test their effect in vivo using a novel reciprocal rat cross. 
The Dlk1-Dio3 locus harbors a set of developmentally regulated genes that show conservation across vertebrates (Edwards et al., 2008). Eutherian mammals including human, rat, and mouse imprint this locus, where the paternally expressed genes are Dlk1, Rtl1, and Dio3, and the maternally expressed genes include non-coding RNAs (ncRNAs), small nucleolar RNAs (snoRNAs) in the rat, and the Gtl2 gene (Royo et al., 2007). Here, we sequenced SD and BN DNA at regions that could potentially regulate Dio3 imprinting. The first candidate region is the Dio3 promoter region, if a hippocampal-specific transcription factor interacts with a polymorphic site. Another region that could influence hippocampal Dio3 allelic expression patterns is the intergenic differentially methylated region (IGDMR) located between $D l k 1$ and $R t l 1$. This region has a series of $\mathrm{CpG}$ sites that are methylated on the paternal allele but unmethylated on the maternal allele. Deletion of $4.1 \mathrm{~kb}$ of the IGDMR in mice results in almost abolished expression of the non-coding transcripts that are normally expressed maternally, and the activation of the maternal allele of the genes that are normally expressed paternally, Dlk1, Rtll, and Dio3 (Lin et al., 2003). Thus, the IGDMR is necessary for the maintenance of maternal silencing for the Dio3 gene and for proper imprinted expression of other transcripts in the locus. We reasoned that if these regions were to harbor BN-SD polymorphisms, their effect on hippocampal allele-specific Dio3 expression could be tested in a new reciprocal F1 rat cross.

\section{MATERIALS AND METHODS ANIMALS}

Animal procedures were approved by the Northwestern University Animal Care and Use Committee and conducted as described previously (Sittig et al., 2011a). Long Evans (LE) and $\mathrm{BN}$ females were crossed to $\mathrm{BN}$ or $\mathrm{LE}$ males, respectively, to generate $\mathrm{F} 1$ reciprocal offspring, $\mathrm{LB}$ and $\mathrm{BL}$, at least 6 offspring/sex/group for fetal and adult analyses. For the fetal study, dams were sacrificed by decapitation on gestational day 21 (G21). Additional pregnant dams were allowed to give birth and rear litters. One or two males and one or two females from each litter were given behavioral tests between 10 and 20 weeks of age. Behavioral testing was staggered with age such that animals were tested in SI at about 10 weeks of age and OFT at 18 weeks of age. Adult animals were sacrificed by decapitation after a 2 week rest after behavioral testing, and their brains were used for allelic expression analyses. Additional brains from littermates were obtained from behaviorally naïve animals. Brain dissections of fetal and adult brains were performed as described (Sittig et al., 2011a).

\section{BEHAVIORAL TESTING}

To test for anxiety behavior, we used two paradigms that have a well-established anxiety component, the open field test (OFT), and a social interaction paradigm. The OFT and social interaction tests $(N=5-10 /$ strain/sex) were conducted on adult rats at $>60$ days of age as described previously (Sittig et al., 2011a, 2012), with $>2$ weeks' rest between tests. For OFT, TSE Videomot 2 software (version 5.75, Bad Homburg, Germany) was used to collect and analyze the data including total distance traveled and time spent in the outer and inner $(50 \mathrm{~cm}$ diameter $)$ regions. Rats were placed in a circular arena $82 \mathrm{~cm}$ in diameter for $10 \mathrm{~min}$ and allowed to freely explore while activity was tracked by the software (Nosek et al., 2008). The arena was lit with a brightness of 60 lux. For social interaction, adult rats were exposed to juvenile stimulus pups of 24-28 days of age and the interaction was video-recorded for $4 \mathrm{~min}$. Adults were individually habituated in a testing cage for $5 \mathrm{~min}$ before beginning the first trial. A trained observer scored olfactory investigation of the adult toward the pup, defined as seconds spent in direct contact.

\section{In silico MAPPING AND GENOMIC ANALYSIS}

We mapped the location of rat Dio3 by using the basic local alignment sequence tool (BLAST) available from the National Center for Biotechnology Information (NCBI). Dio3 has been mapped to Chr 6 random: 1,588,184-1,590,044 in the rat genomic assembly (Rat Genome Database, RGD_v3.4). To define the actual chromosomal location, we calculated the distance in base pairs between the Dio3 transcript and the nearest correctly assembled gene, $P p p 2 r 5 c$, the first non-imprinted gene located $3^{\prime}$ of the Dlk1-Dio3 locus.

A rat homolog to the mouse IGDMR regulatory region was determined by homology to the $4.15 \mathrm{~kb}$ sequence which was deleted in the mouse (Lin et al., 2003). Polymorphic rates between rat strains were determined using SNPlotyper at the Rat Genome Database (http://snplotyper.mcw.edu/). To determine \% polymorphic rates to the outbred SD strain we used the phylogenetic neighbors, SS/JrHsd and SR/JrHsd, and reported a range. A similar approach was used with LE/Han and Le/Stm for the LE rat. The BN strain used was BN/SSNHsd. The phylogenetic relationships among laboratory rat strains were presented previously (Saar et al., 2008). Transcription factor binding sites were predicted using Transcription Element Search System (www.cbil. upenn.edu/cgi-bin/tess/).

\section{Dio3 PYROSEQUENCING PCR AND PYROSEQUENCING}

Pyrosequencing is a reliable method for validating allele-specific expression (Deveale et al., 2012). Methods for Dio3 analyses including RNA extraction, reverse transcription, and PCR and pyrosequencing ( $N=5-10 /$ strain/sex/developmental stage/brain region) are identical to those described (Sittig et al., 2011a). Total RNA extraction was performed using Trizol reagent (Life Technologies, Gaithersburg, MD, USA) according to the manufacturer's protocol. Genomic DNA was removed using the TURBO DNA-free kit (Applied Biosystems, Foster City, CA, USA). DNased RNA (1 ug) was reverse transcribed using the TaqMan Reverse Transcription kit (Applied Biosystems, Branchburg, NJ, USA). Primers for PCR and pyrosequencing were reported previously (Sittig et al., 2011b).

\section{SEOUENCING OF GENOMIC REGIONS}

DNA extraction was performed with standard procedures as described (Sittig et al., 2012). PCR primers were designed using NCBI's Primer Design program. Sequencing primers were designed using the Saccharomyces Genome Database Web Primer program (http://www.yeastgenome.org/cgi-bin/ web-primer). PCR and sequencing primers for Dio3 promoter, as well as the initial sequencing of the rat IGDMR, are described 
in Table 1. The IGDMR was divided into five $\sim 1 \mathrm{~kb}$ sections (I-V) for sequencing purposes. PCR reactions $(N=3 /$ strain $)$ were verified in size (bp) by agarose gel electrophoresis and sequenced by ACGT, Inc. (Wheeling, IL).

\section{STATISTICS}

Data were first analyzed by Two-Way ANOVA using strain, sex, developmental stage, and brain region as factors where applicable. Data were pooled and re-analyzed by $t$-test if there was no interaction and no effect of either strain or sex in the initial analysis, or to test-specific hypotheses. Data are males and females combined unless otherwise noted. Significance indicated on figures represents the result of Bonferroni corrected $t$-tests.

\section{RESULTS}

\section{MAPPING THE DIK1-Dio3 LOCUS IN THE RAT}

The coding sequence and relative position of rat Dio3 at the distal end of the Dlk1-Dio3 locus is known, but the location of Dio3 is currently reported as Chr 6 random: 1,588,184-1,590,044 region in the rat genomic assembly (Rat Genome Database, RGD_v3.4). Here, we mapped the 1859 bp Dio3 coding exon and $3^{\prime}$ UTR to rat Chr 6: 135,030,211-135,032,070. Figure 1 shows a schematic map of the rat Dlk1-Dio3 imprinted locus, with its $3^{\prime}$ boundary designated as the $3^{\prime}$ end of Dio3. Dlk1 has been mapped previously, so the Dlk1-Dio3 imprinted region spans $1.01 \mathrm{Mb}$ on Rat Chr 6: 134,022,016-135,032,070.
Based on our finding that hippocampal Dio3 allelic expression differ between reciprocal crosses SB and BS and exhibited a maternal rather than paternal preference of allelic expression in the $\mathrm{SD}$-mothered (SB) reciprocal cross, we aimed to identify genetic polymorphisms that explain this expression pattern. Several polymorphisms within the Dlk1-Dio3 locus are not candidates for explaining region-specific allelic expression differences. First, a polymorphism between $\mathrm{BN}$ and $\mathrm{SD}$ strains has previously been identified at bp 342 of the Dio3 exon (rs8162867, Figure 1). In our previous reports we have used this synonymous polymorphism to follow allelic expression patterns of Dio3 in reciprocal BN-SD crosses (Sittig et al., 2011a,b). This G/C rs8162867 SNP could affect transcription differently across brain regions only if a hippocampal-specific transcription factor were to bind there. We searched for such binding sites across this SNP for both the " $\mathrm{C}$ " and " $\mathrm{G}$ " alleles, and found one hit for the " $\mathrm{G}$ " allele, the negative glucocorticoid response element (R01813). If glucocorticoid receptor does bind to the $G$ allele, this may explain the hippocampal-specific repression of the paternal Dio3 expression in SB and LB animals only if hippocampal-specific accessory factors allow binding in the hippocampus but not frontal cortex.

A second potentially regulatory region is the IGDMR. It was demonstrated that the maternal IGDMR is necessary for maintenance of maternally expressed non-coding transcript expression, and for the repression of the maternal copy of paternally expressed genes in the locus, including Dio3 (Lin et al., 2003). Genetic differences at the IGDMR would likely affect

Table 1 | PCR and sequencing primers for IGDMR and Dio3 promoter.

\begin{tabular}{|c|c|c|c|}
\hline Region & Section $\left(5^{\prime}-3^{\prime}\right)$ & Sequence $5^{\prime}-3^{\prime}$ & bp product \\
\hline \multirow[t]{20}{*}{ IGDMR } & I & F: CCTCTAGGCTTTACTCGTGGGGG & 1066 \\
\hline & & R: AGGCGCTTGGCTCCTACCGT & \\
\hline & I-sequencing & F: GTAGGTAGTACACCATCC & - \\
\hline & & R: AGTGTACCACGTAGCTGT & \\
\hline & $\|$ & F: CGCTTCGTGGCGCAAACACAT & 1000 \\
\hline & & R: CGCGGCATTGTGAACCGTGG & \\
\hline & Il-sequencing & F: CGTGTACTATGTATGGTA & - \\
\hline & & R: CGTTTGTCTGGAGTACAC & \\
\hline & III & F: TTGGGCCAGTCCCAGGGCT & 960 \\
\hline & & R: GACTCCTCTAGGATCCCCGGAGC & \\
\hline & III-sequencing & F: TACTGTGCACAAGGACTG & - \\
\hline & & R: TTATGGGTCTTCCTTTTC & \\
\hline & IV & F: CCTCCTCCCTAGGTTGCCCTTAGTA & 1030 \\
\hline & & R: TTGGCCAAGTGAGAGACATCTGCAA & \\
\hline & IV-sequencing & F: ATCCTCATCTTCAAATAC & - \\
\hline & & R: AACAAGTAGGTTCAGGTG & \\
\hline & V & F: TAGGAATGAGAGAGCCCTACCCCA & 1059 \\
\hline & & R: GCAAGCCTGCATGTATGTGCAGAG & \\
\hline & V-sequencing & F: ACACCTGAACCTACTTGTT & - \\
\hline & & R: AGCTAGCTTGTTTGCAGG & \\
\hline \multirow[t]{4}{*}{ Dio3 promoter } & -452 to Dio3 & F: CGCGGGAAGCGAACCGGAG & 681 \\
\hline & & R: GGTCAGGATGACGACGGCGC & \\
\hline & Sequencing & F: TGCGGCCGCCGGGGAACTCA & - \\
\hline & & R: AGGAAATGCTTGCGGATG & \\
\hline
\end{tabular}






imprinted expression at the entire locus, and for Dio3, would affect it across tissues rather than producing a hippocampalspecific effect. We deemed it unlikely that the IGDMR harbors a mutation in the $\mathrm{SD}$ or $\mathrm{BN}$ strain for this reason and because neither strain displays any of the severe developmental phenotypes associated with IGDMR mutations and/or disruption of imprinting at the Dlk1-Dio3 locus (Lin et al., 2003, 2007; Kagami et al., 2010). Nevertheless, we mapped and sequenced the rat IGDMR as identified based on homology to the mouse IGMDR (PCR and sequencing primers in Table 1). This $4.1 \mathrm{~kb}$ region showed no polymorphisms between SD and BN strains. A schematic of mapping the putative rat IGDMR (Chr 6: 134,022,016-135,032,070) is given in Figure 2.

\section{FOUR NOVEL SNPS WITHIN THE PROXIMAL Dio3 PROMOTER}

A strong candidate region that could influence Dio3 allelic expression patterns region-specifically is the Dio3 promoter. For example, if there were a hippocampal-specific transcription factor binding site or alternative promoter, a polymorphism could affect hippocampal transcription factor binding asymmetrically in the two reciprocal crosses. At least four differentially sized Dio3 transcripts in rat brain (Tu et al., 1999) suggest the possibility of a brain region-specific promoter(s). Promoter activity in vitro has

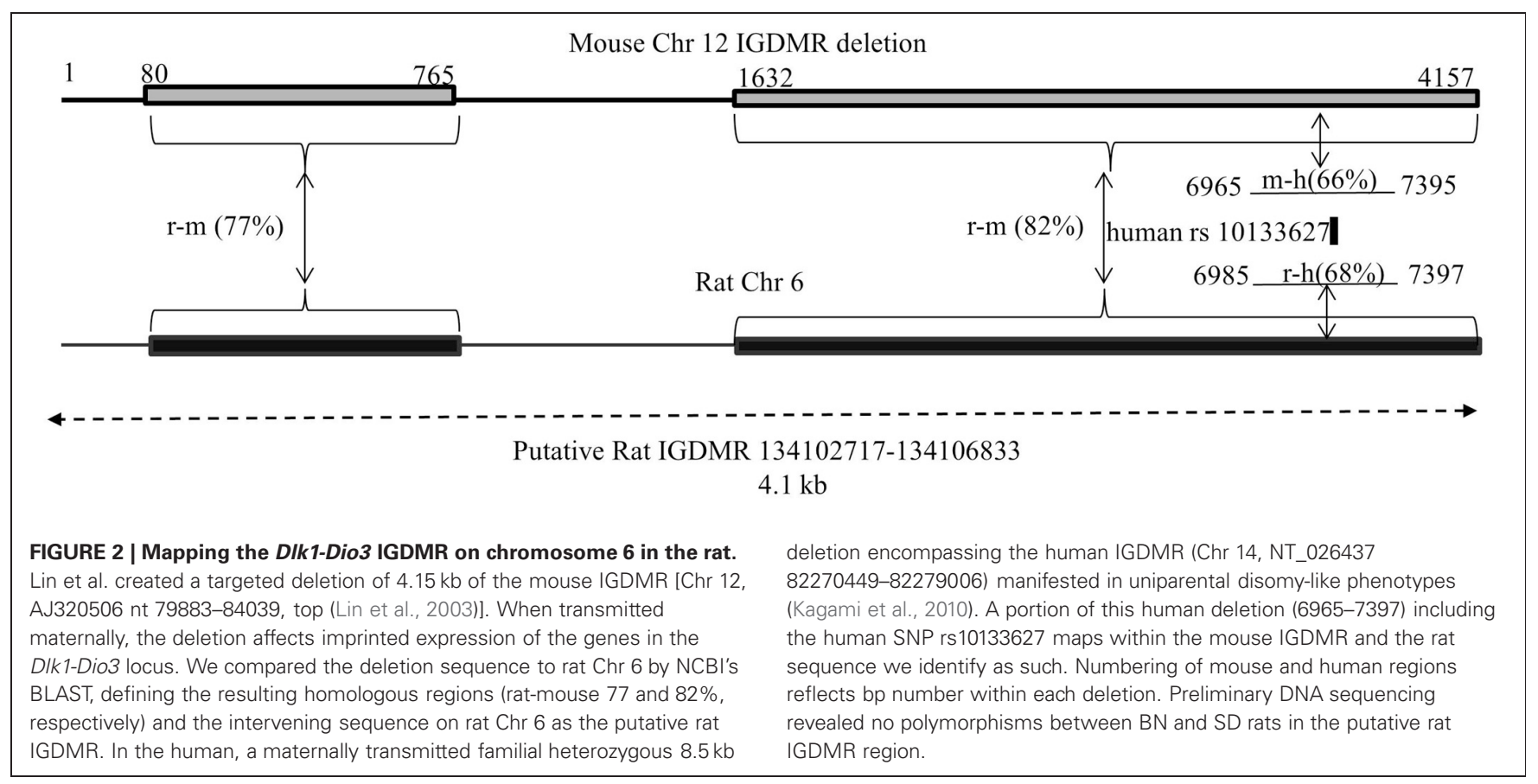


been shown for $1.8 \mathrm{~kb}$ upstream from Dio3 in mouse and human with the strongest promoter activity occurring $\sim 450$ bp upstream from the transcription start site (Hernandez and St. Germain, 2003). This putative promoter region $(1.8 \mathrm{~kb}-0 \mathrm{bp}$ upstream of the Dio3 start site) exhibits $92 \%$ identity between mouse and rat, and the 450 bp most proximal to Dio3 exhibits $96 \%$ identity and 74\% GC content. Four BN/SD polymorphisms were identified within the most proximal subregion of the putative rat Dio3 promoter, $-360,-335,-305$, and -100 bp upstream from the Dio3 coding sequence (Figures 3A-D).

\section{LE RECAPITULATES THE SD GENOTYPE IN THE Dio3 EXON AND ITS PROMOTER}

To determine whether there is a genetic underpinning for brain region-specific variations in Dio3 allelic expression and its consequences, we evaluated Dio3 allelic expression in a new reciprocal cross. If SD-BN genetic polymorphisms within the Dlk1Dio3 imprinted region are causative toward variant Dio3 allelic expression observed in SB vs. BS rats, then we should observe a similar pattern of Dio3 expression in a different reciprocal cross where the SD has been replaced with a genetically similar strain. Since BN is phylogenetically divergent from other

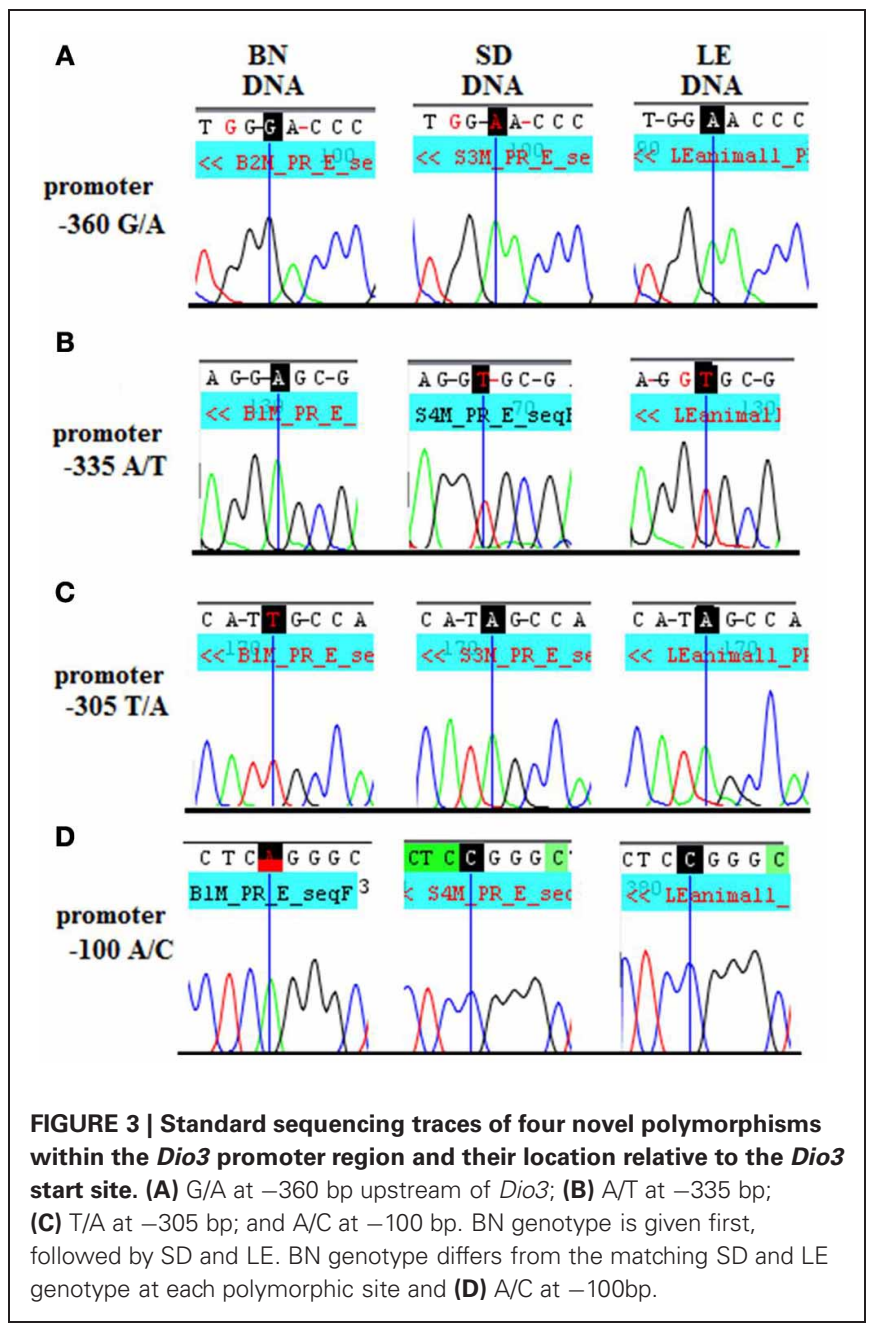

laboratory rat strains (Saar et al., 2008), we utilized the BN in a new cross. For the other strain we wanted to maintain the SD haplotype in the Dio3 promoter but otherwise introduce genetic polymorphisms. We genotyped several candidate strains at several regions (data not shown), including the LE, which is phylogenetically close to the SD. The adult male LE rat also has similar peripheral TSH and T4 levels as the SD (Pekary et al., 1984), indicating there are not major genetic differences in thyroid function between these strains. The LE rat matched the SD genotype at all regions and all polymorphic sites identified in the Dio3 region (Figures 3A-D), yet exhibits 29-31\% polymorphic rate to SD-like strains. Thus, the LE is a genetically divergent strain in which many loci would be polymorphic with the SD, but the Dio3 promoter region and exon are the same.

\section{LB-BL RECIPROCAL CROSSES REPLICATE ALLELIC Dio3 EXPRESSION PATTERNS IN HIPPOCAMPUS WITH DIFFERENCES IN ANXIETY-RELATED BEHAVIOR}

As expected, BL animals exhibited slightly paternal to biallelic expression in the fetal frontal cortex and hippocampus and biallelic expression in the adult hippocampus, confirming the results seen previously in the BS reciprocal cross (Figure 4). BL Dio3 expression in adult frontal cortex averaged slightly less than $50 \%$ paternal, but this appears to be an artifact of higher variability. In contrast, LB frontal cortex and hippocampus showed

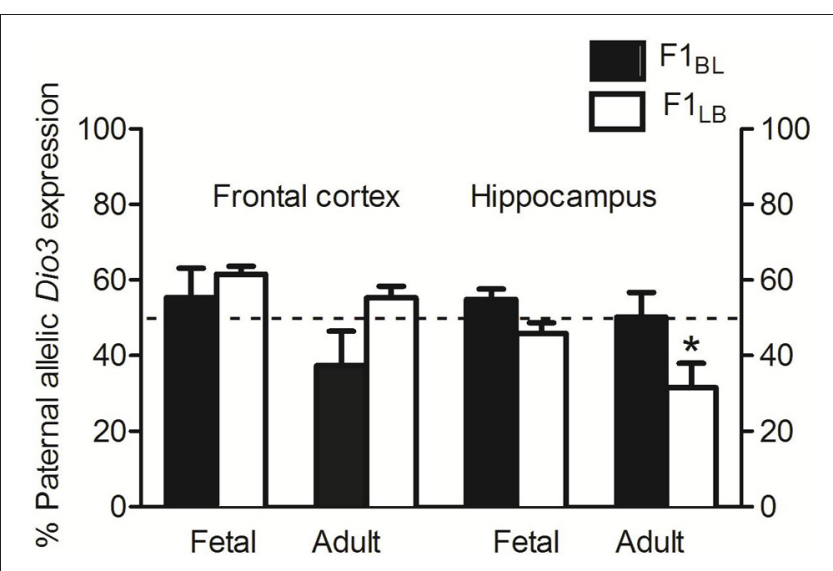

FIGURE 4 | Hippocampal-specific maternal Dio3 allelic expression in LB reciprocally crossed offspring with anxiety-related behavior. LB animals exhibit differential Dio3 allelic expression in hippocampus only, while $B L$ offspring show no significant deviations from the expected allelic expression pattern of Dio3 in either brain region. Data represent means of individual animals' Dio3 allelic expression values determined by quantitative pyrosequencing of cDNAs from each brain region. Results from animals that underwent behavioral testing did not differ from naïve animals. $N=12-14$ individual fetal frontal cortex/strain, and $N=12-14$ individual fetal hippocampal regions/strain. $N=12-13$ individual adult frontal cortex/strain, and $N=13-15$ individual adult hippocampal regions/strain. Data were first analyzed by Two-Way ANOVA using strain, sex,

developmental stage, and brain region as factors where applicable. Data were pooled and re-analyzed by $t$-test if there was no interaction and no effect of either strain or sex in the initial analysis, or to test specific hypotheses. Data are males and females combined unless otherwise noted. ${ }^{*} p<0.05$ vs. frontal cortex, Bonferroni corrected. 
divergent Dio3 expression patterns (Figure 4). There was a nonsignificant trend toward an interaction between strain and brain region for Dio3 allelic expression in fetal frontal cortex and hippocampus $\left[F_{(1,49)}=2.6, p=0.11\right]$, and adults showed a significant interaction $\left[F_{(1,49)}=7.8, p<0.01\right]$. Specifically, LB adults exhibited preferentially maternal Dio3 expression in the hippocampus but preferentially paternal Dio3 expression in the frontal cortex (Figure 4). These findings recapitulate the unique maternal preference for Dio3 allelic expression that was reported in SB animals only in the hippocampus (Sittig et al., 2011a).

Previously, SB animals showing maternal hippocampal Dio3 expression exhibited anxiety-like behaviors compared to the reciprocal BS cross. We therefore tested BL and $\mathrm{LB}$ animals on a well-established measure for anxiety, the OFT. LB males and females spent more time in the outer, anxiety-reducing portion of the circular OFT arena compared to BL animals $\left[t_{(25)}=2.6\right.$, $p<0.05$, data not shown], suggesting a higher level of anxiety. Figure 5A shows that LB males only spent less time in the inner, anxiogenic region of the arena $\left[t_{(12)}=2.6, p<0.05\right.$; hypothesis testing due to trends of strain and sex in the ANOVA], suggesting that the anxiety phenotype was more pronounced in the LB males than in LB females. LB animals also traveled less distance than $\mathrm{BL}\left[F_{(1,23)}=8.7, p<0.01\right]$ (Figure 5B), a phenotype which is known to occur concomitant with other anxiety-like behaviors in rats (Naqvi et al., 2012; Rosenfeld and Weller, 2012). Finally, we asked whether the social anxiety exhibited by SB was also present in LB of the current study. We found that LB males in fact displayed more social investigation than $\mathrm{BL}$ [interaction of sex and strain: $F_{(1,28)}=7.1, p<0.05$ ] (Figure 5C). This finding indicates that it is very unlikely that the LB animals' hypoactivity in the OFT was due to a motor deficit.

\section{DISCUSSION}

The experiment presented here is an approximated parent-oforigin specific congenic experiment which confirms the effect of the SD genotype at the Dio3 promoter when inherited maternally. Our original reciprocal cross parental strains, BN and SD, exhibit a polymorphic rate of $\sim 62 \%$. Since the SD-LE exhibit $\sim 31 \%$ polymorphic rate, there are significant genetic differences between the SB-BS vs. LB-BL reciprocal crosses. Furthermore,

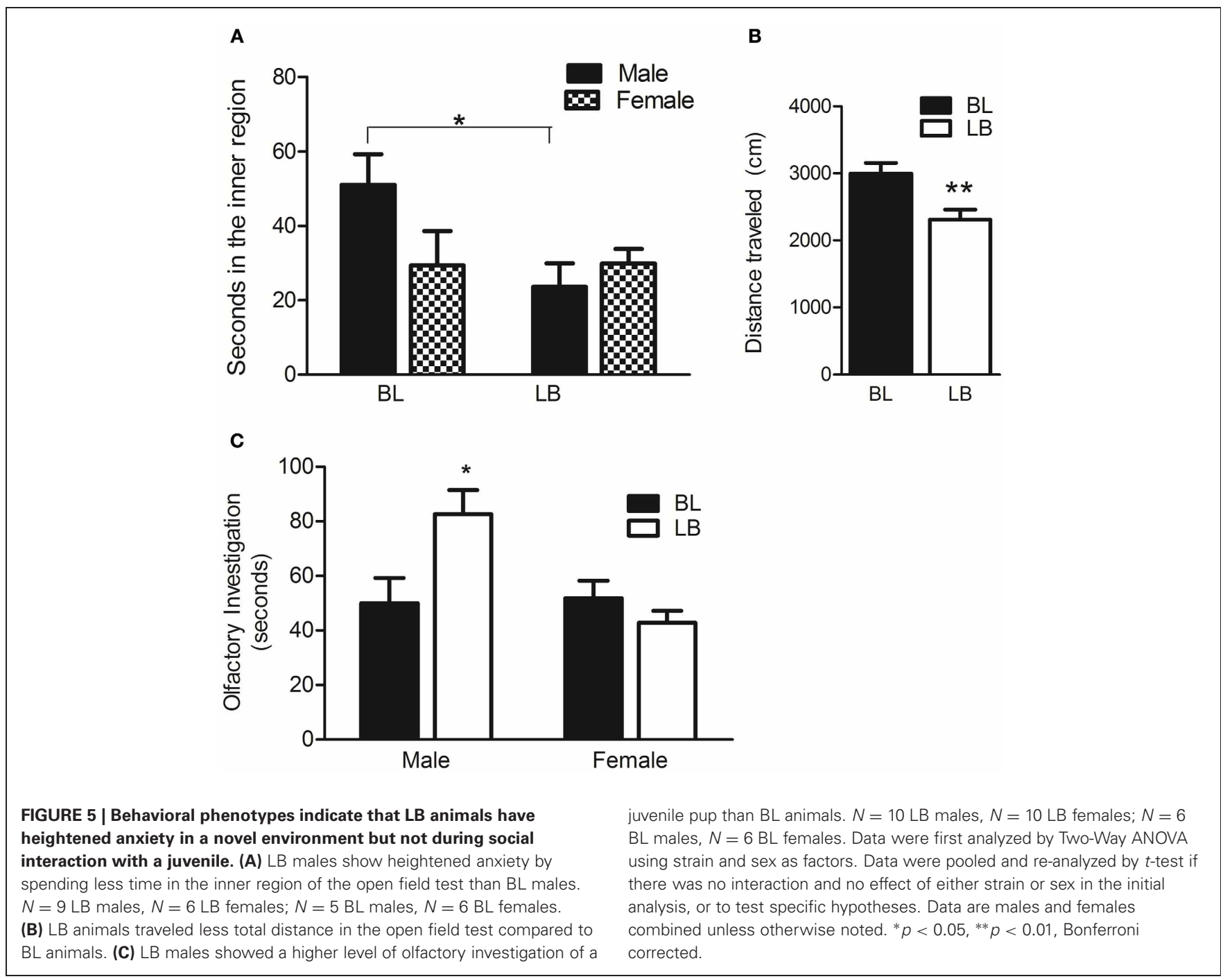


the $\mathrm{BN}-\mathrm{LE}$ polymorphic rate is $51-52 \%$, more than $10 \%$ less than $\mathrm{BN}-\mathrm{SD}$. Here we have taken advantage of the fact that the polymorphisms in SB-BS vs. LB-BL reciprocal crosses do not differ in the Dio3 promoter region but do differ elsewhere. There are several potential mechanisms of the hippocampal-specific Dio3 gene expression, including differential expression of alternative Dio3 transcripts, differential use of alternative promoters, and polymorphisms in cis- or trans-acting transcription factor binding sites or transcription factors themselves. Whichever of these is true, the eventual elucidation of the correct mechanism may involve cis-polymorphisms close to the Dio3 gene itself. The current work reveals the specific polymorphisms at the Dio3 promoter region which could be mechanistically involved in the strain- and brain region- specific regulation of gene expression. Mechanistic proof of the Dio3 promoter polymorphisms' effects could be carried out in primary cultures from cortex and hippocampus of our reciprocal crosses.

Our data is consistent with the idea that Dio3 is one of many genes which by small variations in its expression, affects brain circuits that control complex, multigenic behaviors including anxiety-like behavior. LB animals were less active than BL in the OFT, which could be interpreted as anxiety-related behavioral inhibition in a novel situation. However, hypoactivity alone is not likely to account for the fact that LB males spent less time in the center of the OFT since LB females showed less total activity but spent the same time in the center of the maze as BL females. In addition, LB males interacted more with juvenile stimulus pup in social interaction, a behavior that is highly dependent on intact mobility. Additional behavioral assays would be required to firmly associate Dio3 expression with anxiety-like behavior, but our current data indicate the proof-of-concept that LB rats behaved differently from BL rats on a classic, stable measure of anxiety: time spent in the periphery of the open field.

\section{REFERENCES}

Davies, W., Isles, A. R., and Wilkinson, L. S. (2005). Imprinted gene expression in the brain. Neurosci. Biobehav. Rev. 29, 421-430.

Deveale, B., Van Der Kooy, D., and Babak, T. (2012). Critical evaluation of imprinted gene expression by RNA-Seq: a new perspective. PLoS Genet. 8:e1002600. doi: 10.1371/journal.pgen. 1002600

Edwards, C. A., Mungall, A. J., Matthews, L., Ryder, E., Gray, D. J., Pask, A. J., et al. (2008). The evolution of the DLK1-DIO3 imprinted domain in mammals. PLoS Biol. 6:e135. doi: 10.1371/journal.pbio.0060135

Gregg, C., Zhang, J., Weissbourd, B., Luo, S., Schroth, G. P., Haig, D., et al. (2010). High-resolution analysis of parent-of-origin allelic expression in the mouse brain. Science 329, 643-648.

Hernandez, A., and St. Germain, D. L. (2003). Activity and response to

In this report, we first mapped and sequenced genomic regions that likely contribute to an unexpected and novel finding, aberrant allelic expression of the thyroid hormone metabolizing gene Dio3 in the hippocampus of one reciprocal rat cross. We confirmed this novel pattern of expression using a different reciprocal cross harboring the same genetic differences in the promoter region of Dio3, indicating that parent-of-origin Dio3 expression in the hippocampus is of genetic origin, and confirmed the co-occurrence of anxiety-like behavior. We cannot exclude the possibility that other SNPs within the Dlk1-Dio3 locus (e.g., in the ncRNA transcripts found there) and/or outside the locus are important for the findings about allelic expression patterns reported herein. Polymorphisms across the genome and not only in and around Dio3 are likely to contribute to the behavioral differences between reciprocal crosses, but these effects are at a minimum in the current study, since strains being compared are two reciprocal F1 crosses and are therefore genetically identical. In humans, parent-of-origin specific genetic effects modulating brain function are a possibility that is beginning to undergo exploration. Should human orthologs for polymorphisms within Dio3 be found, they could be important in explaining differences in neuropsychiatric vulnerability and cognitive functioning. In the future, polymorphisms that lead to brain region-specific allelic gene expression, as well as the genetic $\times$ epigenetic interactive mechanisms of this specificity, will need to be elucidated in both rodent models and humans.

\section{ACKNOWLEDGMENTS}

The authors thank Dr. Laura Herzing for thoughtful discussions and sequencing of the exonic SNP in Dio3. This work was funded by RO1 AA017978 to Eva E. Redei and F31 AA018251 to Laura J. Sittig.

control region. Development 134, 417-426.

serum of the mammalian thyroid hormone deiodinase 3 gene promoter: identification of a conserved enhancer. Mol. Cell. Endocrinol. 206, 23-32.

Kagami, M., O’Sullivan, M. J., Green, A. J., Watabe, Y., Arisaka, O., Masawa, N., et al. (2010). The IG-DMR and the MEG3-DMR at human chromosome 14q32.2: hierarchical interaction and distinct functional properties as imprinting control centers. PLoS Genet. 6:e1000992. doi: 10.1371/ journal.pgen.1000992

Kernohan, K. D., and Berube, N. G. (2010). Genetic and epigenetic dysregulation of imprinted genes in the brain. Epigenomics 2, 743-763.

Lin, S. P., Coan, P., Da Rocha, S. T., Seitz, H., Cavaille, J., Teng, P. W., et al. (2007). Differential regulation of imprinting in the murine embryo and placenta by the Dlk1-Dio3 imprinting
Lin, S. P., Youngson, N., Takada, S., Seitz, H., Reik, W., Paulsen, M., et al. (2003). Asymmetric regulation of imprinting on the maternal and paternal chromosomes at the Dlk1-Gtl2 imprinted cluster on mouse chromosome 12. Nat. Genet. 35, 97-102.

Naqvi, F., Haider, S., Batool, Z., Perveen, T., and Haleem, D. J. (2012). Sub-chronic exposure to noise affects locomotor activity and produces anxiogenic and depressive like behavior in rats. Pharmacol. Rep. 64, 64-69.

Nosek, K., Dennis, K., Andrus, B. M., Ahmadiyeh, N., Baum, A. E., Woods, L. C., et al. (2008). Context and strain-dependent behavioral response to stress. Behav. Brain Funct. 4:23. doi: 10.1186/17449081-4-23

Pekary, A. E., Carlson, H. E., Yamada, T., Sharp, B., Walfish, P. G., and Hershman, J. M. (1984). Thyrotropin-releasing hormone levels decrease in hypothalamus of aging rats. Neurobiol. Aging 5, 221-226.

Rosenfeld, A., and Weller, A. (2012). Behavioral effects of environmental enrichment during gestation in WKY and Wistar rats. Behav. Brain Res. 233, 245-255.

Royo, H., Basyuk, E., Marty, V., Marques, M., Bertrand, E., and Cavaille, J. (2007). Bsr, a nuclearretained RNA with monoallelic expression. Mol. Biol. Cell 18, 2817-2827.

Saar, K., Beck, A., Bihoreau, M. T., Birney, E., Brocklebank, D., Chen, Y., et al. (2008). SNP and haplotype mapping for genetic analysis in the rat. Nat. Genet. 40, 560-566.

Sittig, L. J., Herzing, L. B., Shukla, P. K., and Redei, E. E. (2011a). Parent-of-origin allelic contributions to deiodinase-3 expression 
elicit localized hyperthyroid milieu in the hippocampus. Mol. Psychiatry 16, 786-787.

Sittig, L. J., Shukla, P. K., Herzing, L. B., and Redei, E. E. (2011b). Strain-specific vulnerability to alcohol exposure in utero via hippocampal parent-of-origin expression of deiodinase-III. FASEB J. 25, 2313-2324.

Sittig, L. J., Herzing, L. B., Xie, H., Batra, K. K., Shukla, P. K., and Redei, E. E. (2012). Excess folate during adolescence suppresses thyroid function with permanent deficits in motivation and spatial memory. Genes Brain Behav. 11, 193-200.

Tu, H. M., Legradi, G., Bartha, T., Salvatore, D., Lechan, R. M., and Larsen, P. R. (1999). Regional expression of the type 3 iodothyronine deiodinase messenger ribonucleic acid in the rat central nervous system and its regulation by thyroid hormone. Endocrinology 140, 784-790.
Conflict of Interest Statement: The authors declare that the research was conducted in the absence of any commercial or financial relationships that could be construed as a potential conflict of interest.

Received: 02 August 2012; accepted: 29 November 2012; published online: 14 December 2012.

Citation: Sittig LJ and Redei EE (2012) Novel polymorphisms within the Dlk1Dio3 imprinted locus in rat: a putative genetic basis for strain-specific allelic gene expression. Front. Gene. 3:296. doi 10.3389/fgene.2012.00296

This article was submitted to Frontiers in Behavioral and Psychiatric Genetics, a specialty of Frontiers in Genetics.

Copyright (c) 2012 Sittig and Redei. This is an open-access article distributed under the terms of the Creative Commons Attribution License, which permits use, distribution and reproduction in other forums, provided the original authors and source are credited and subject to any copyright notices concerning any third-party graphics etc. 